\title{
Obtenção de Piches Mesofásicos em Dois Estágios a partir de Piche de Petróleo
}

\author{
Carlos H. M. C. Dutra \\ Centro Tecnológico do Exército, RJ, Instituto de Macromoléculas Professora Eloisa Mano, UFRJ \\ Luiz D. de Castro \\ Centro Tecnológico do Exército, $R J$ \\ Cristina T. Andrade \\ Instituto de Macromoléculas Professora Eloisa Mano, UFRJ
}

\begin{abstract}
Resumo: A obtenção de piches mesofásicos para a produção de fibras de carbono de alta performance foi investigada por meio de tratamento térmico de três piches de petróleo, em dois estágios em um reator com agitação magnética e capacidade de 700 g. O primeiro estágio consistiu de um pré-tratamento sob agitação e atmosfera de nitrogênio a 0,9 MPa, a 390,410 e $430{ }^{\circ} \mathrm{C}$, durante três horas. No segundo estágio, o tratamento térmico foi realizado por mais três horas, sob pressão atmosférica. Durante os experimentos, amostras foram recolhidas e analisadas por meio de medidas de solubilidade em tolueno e quinolina, ponto de amolecimento e o percentual de mesofase. Foi verificado que, para todos os piches produzidos, nas temperaturas de 390 e $410{ }^{\circ} \mathrm{C}$ a variação dos parâmetros físico-químicos foi muito pequena e que os tratamentos térmicos no patamar de $430^{\circ} \mathrm{C}$ produziram piches com pontos de amolecimento acima de $300{ }^{\circ} \mathrm{C}$, e percentual de mesofase próximo a $70 \%$.
\end{abstract}

Palavras-chave: Piche de petróleo, pirólise, piche mesofásico.

\section{Preparation in Two Stages of Mesophase Pitches from Petroleum Pitch}

Abstract: Mesophase pitches, precursors of high-performance carbon fibers, were prepared from petroleum pitch by a twostage heat treatment, in a reactor capable of heat treating $700 \mathrm{~g}$ of petroleum pitch under stirring. The process consisted of a pretreatment under pressure at 390,410 and $430{ }^{\circ} \mathrm{C}$ under nitrogen atmosphere, at $0.9 \mathrm{MPa}$, for three hours, as the first stage, followed by another heat treatment under atmospheric pressure, as the second stage. To study the properties of the petroleum pitch samples, during the experiment, they were removed from the system. Quinoline insolubles, toluene insolubles, mesophase content and softening point of the samples were used to follow the pyrolysis process. The results revealed slight variations in the properties for the pitches produced at 390 and $410{ }^{\circ} \mathrm{C}$. However, the pitch samples produced at $430{ }^{\circ} \mathrm{C}$ had softening points higher than $300{ }^{\circ} \mathrm{C}$, and mesophase contents of around $70 \%$.

Keywords: Petroleum pitch, pyrolysis, mesophase pitch.

\section{Introdução}

Os piches são matérias-primas ricas no elemento carbono e essenciais para a produção de uma série de materiais. Eles têm sido usados como impregnantes para a fabricação de eletrodos de grafite (com aplicação na fabricação de aço), aglutinantes para a fabricação de pasta de eletrodos Soderberg (utilizados na fabricação do alumínio), na produção de coque para a indústria siderúrgica e de alumínio, na produção de grafites especiais largamente empregados da indústria biomédica, nos reatores nucleares e para a produção de fibras de carbono de alto módulo de Young (alta rigidez) e fibras de carbono de baixo custo e de aplicação geral $^{[1,2]}$.

Até recentemente, a maioria dessas aplicações industriais era suprida pelos piches oriundos do alcatrão da hulha (gerado nas coquerias das usinas siderúrgicas, a partir do carvão mineral). No entanto, esse material é extremamente tóxico e altamente poluente. Devido a isso, na última década, teve início um movimento de incentivo à substituição do piche do alcatrão da hulha pelo piche oriundo do petróleo.

O piche mesofásico pode ser obtido tanto a partir do piche de alcatrão, como do piche de petróleo. O piche de petróleo é produzido a partir do óleo decantado, o qual consiste do resíduo aromático resultante do craqueamento catalítico do petróleo pesado. O piche de alcatrão apresenta vantagens no que diz respeito à preparação de piches mesofásicos por ser mais rico em hidrocarbonetos aromáticos, que facilitam a formação da mesofase. Por outro lado, o piche de petróleo apresenta-se isento de insolúveis primários em quinolina, os quais não favorecem a formação da mesofase ${ }^{[3]}$.

Autor para correspondência: Cristina T. Andrade, Grupo de Polímeros Hidrossolúveis, Instituto de Macromoléculas Professora Eloisa Mano, UFRJ, CT, bloco J, Caixa Postal 68525, CEP: 21945-970, Rio de Janeiro, RJ, Brasil. E-mail: ctandrade@ima.ufrj.br 
O uso generalizado do piche mesofásico para produção de fibras de carbono de alta performance apresenta vários problemas, tais como a preparação de um piche capaz de ser fiado $^{[4,5]}$. Alguns autores ${ }^{[6,7]}$ asseguraram que a remoção eficiente de voláteis, durante a preparação, produziria um piche mesofásico fiável a temperaturas moderadamente elevadas. Entretanto, esse procedimento eliminaria a chance de as moléculas mais leves condensarem-se, o que levaria a baixos rendimentos de mesofase, de cerca de 20 a $30 \%{ }^{[8]}$, e elevaria o custo de fabricação da fibra.

Para aumentar o rendimento da produção da mesofase, Park ${ }^{[8]}$ estudou a preparação do piche mesofásico em dois estágios, a partir do óleo decantado oriundo do resíduo do craqueamento catalítico do petróleo (RARO). Os dois estágios consistem de condensação pressurizada, no primeiro estágio, seguido de um segundo estágio, sob vácuo. A condensação sob pressão permitiu que as moléculas leves transformassemse em formadores da mesofase (mesógenos) e que a condensação excessiva das moléculas mais pesadas pudesse ser minimizada. Park ${ }^{[8]}$ concluiu que o tratamento térmico sob pressão, no primeiro estágio, seguido da carbonização sob vácuo, no segundo estágio, mostrou-se muito efetivo para aumentar o rendimento do piche fiável, com 100\% de anisotropia, a partir do RARO. Os dois estágios de preparação produziram um piche com ponto de amolecimento ligeiramente mais baixo, o que facilita a fiação. De acordo com o autor, o tratamento térmico sob pressão teria decomposto quase que completamente as frações parafínicas do RARO, produzido desalquilação dos alquil-aromáticos e condensação dos componentes aromáticos, especialmente das frações mais leves. Portanto, essas reações levaram a uma distribuição mais homogênea das espécies presentes e contribuíram para aumentar o rendimento de moléculas mesogênicas.

$\mathrm{Oh}^{[9]}$ investigou a preparação de piche mesofásico em dois estágios, a partir do piche de alcatrão. O primeiro estágio consistiu de tratamento térmico do piche original sob pressão. No segundo estágio, o tratamento térmico foi realizado sob pressão ambiente, com borbulhamento de nitrogênio, e aquecimento na faixa entre 420 e $500{ }^{\circ} \mathrm{C}$. Segundo o autor, o tratamento sob pressão inibiu a volatilização dos componentes de baixa massa molar, os quais, de outra forma, eram removidos do sistema, devido ao baixo ponto de ebulição, e induziu sua condensação. Por outro lado, esses componentes de baixa massa molar formaram uma fase solvente de menor viscosidade no sistema e, portanto, evitaram uma rápida condensação dos componentes de maior massa molar. O autor concluiu que a condensação das espécies componentes de baixa massa molar foi favorecida a $420{ }^{\circ} \mathrm{C}$, e que o craqueamento térmico dos componentes de alta massa molar, bem como a sua condensação, ocorreu a $450{ }^{\circ} \mathrm{C}$. Acima de $450{ }^{\circ} \mathrm{C}$, a reação de condensação das espécies de alta massa molar passou a constituir-se na reação dominante.

No presente trabalho, a obtenção de piches mesofásicos para a produção de fibras de carbono de alta performance foi investigada por meio de tratamento térmico de um piche de petróleo, em dois estágios. O primeiro estágio consistiu de um pré-tratamento sob agitação e atmosfera de nitrogênio a $0,9 \mathrm{MPa}$, a 390,410 e $430{ }^{\circ} \mathrm{C}$, durante três horas. No segundo estágio, o tratamento térmico foi realizado por mais três horas, sob pressão atmosférica. Durante os experimentos, amostras foram recolhidas e analisadas por meio de medidas de densidade, ponto de amolecimento, valor de coqueificação e solubilidade em solventes.

\section{Experimental}

\section{Material}

Como matéria-prima, foram usados três piches isotrópicos, obtidos por meio de destilação de três resíduos aromáticos (RAROs) do craqueamento catalítico de petróleo, fornecidos pela Petrobras. A escolha desses piches isotrópicos foi baseada principalmente no elevado caráter aromático e no baixo percentual de enxofre. A Tabela 1 mostra um resumo das características físico-químicas dos piches isotrópicos e os resultados de análise elementar. São incluídos valores de massa específica, o ponto de amolecimento (PA), o valor de coqueificação (VC), os insolúveis em tolueno (IT) e os insolúveis em quinolina (IQ).

\section{Métodos}

\section{Obtenção de amostras de piches mesofásicos}

Para cada uma das reações, foram usados $700 \mathrm{~g}$ de piche isotrópico. Os piches foram tratados em um reator com agitador magnético a $400 \mathrm{rpm}$ e submetido a três patamares de temperatura de tratamento térmico, 390,410 e $430{ }^{\circ} \mathrm{C}$. O procedimento experimental pode ser resumido como se segue. Sob atmosfera de nitrogênio (vazão de $0,1 \mathrm{Nm}^{3} / \mathrm{h}$ ), o material era aquecido até o primeiro patamar de temperatura reacional, quando uma amostra era coletada. A saída de voláteis do reator era, então, fechada e a pressão elevada a 0,9 MPa. Es-

Tabela 1. Propriedades físico-químicas e análise elementar dos piches isotrópicos.

\begin{tabular}{cccc}
\hline \multirow{2}{*}{ Ensaios } & \multicolumn{3}{c}{ Piches isotrópicos $^{\mathbf{a}}$} \\
\cline { 2 - 4 } & $\mathbf{A}$ & $\mathbf{B}$ & $\mathbf{C}$ \\
\hline P. de amolecimento $\left({ }^{\circ} \mathrm{C}\right)$ & 104,0 & 107,7 & 103,0 \\
Massa específica $\left({\left.\mathrm{g} . c{ }^{-3}\right)}^{-3}\right)$ & 1,257 & 1,232 & 1,227 \\
Valor de coqueificação (\%) & 55 & 57 & 53 \\
Insolúveis em tolueno (\%) & 15,2 & 19,2 & 12,5 \\
Insolúveis em quinolina (\%) & 2,7 & 3,3 & 1,6 \\
$\mathrm{C}(\%)$ & 90,66 & 91,38 & 90,27 \\
$\mathrm{H}(\%)$ & 5,25 & 5,28 & 5,53 \\
$\mathrm{~N}(\%)$ & 0,38 & 0,70 & 0,72 \\
$\mathrm{~S}(\%)$ & 0,79 & 0,85 & 0,77 \\
$\mathrm{O}(\%)$ & 2,92 & 1,79 & 2,71 \\
$\mathrm{C} / \mathrm{H}$ & 1,449 & 1,452 & 1,370 \\
\hline
\end{tabular}

${ }^{\mathrm{a}} \mathrm{A}, \mathrm{B}$ e C são piches isotrópicos de petróleo com teores elevados de aromáticos e baixos teores de enxofre. 
sas condições reacionais eram mantidas por três horas. Após esse período, a pressão era reduzida lentamente e, sob pressão atmosférica, uma nova amostra era coletada. Em seguida, novas amostras eram coletadas a cada hora, sempre sob atmosfera de nitrogênio. As amostras eram resfriadas rapidamente em uma vasilha com água e enviadas para análise.

\section{Determinação do ponto de amolecimento}

Os pontos de amolecimento foram determinados em equipamento Mettler-Toledo, modelo FP900, de acordo com a norma ASTM D 3104-87.

\section{Determinação de insolúveis em tolueno}

Os insolúveis em tolueno (IT) foram determinados de acordo com a norma ASTM D 4312.

\section{Determinação de insolúveis em quinolina}

Os insolúveis em quinolina (IQ) foram calculados por meio de correlações publicadas em relatórios internos do centro tecnológico do Exército e da Petrobras, a partir dos teores de insolúveis em 1-metil-2-pirrolidinona.

\section{Determinação do teor de mesofase}

O teor de mesofase foi determinado a partir da separação de fases, obtida após centrifugação a $3600 \mathrm{rpm}, 320^{\circ} \mathrm{C}$, durante 60 minutos conforme reportado por Dutra et al. ${ }^{[10]}$. As frações isotrópicas e anisotrópicas foram quantificadas em balança de precisão.

\section{Resultados e Discussão}

Para o piche isotrópico B, a Figura 1 mostra que o efeito da temperatura foi significativo e influenciou positivamente o teor de insolúveis em tolueno e em quinolina. O aumento do teor de insolúveis, observado após o abaixamento da pressão, pode ter sido causado pelo abaixamento da pressão, após

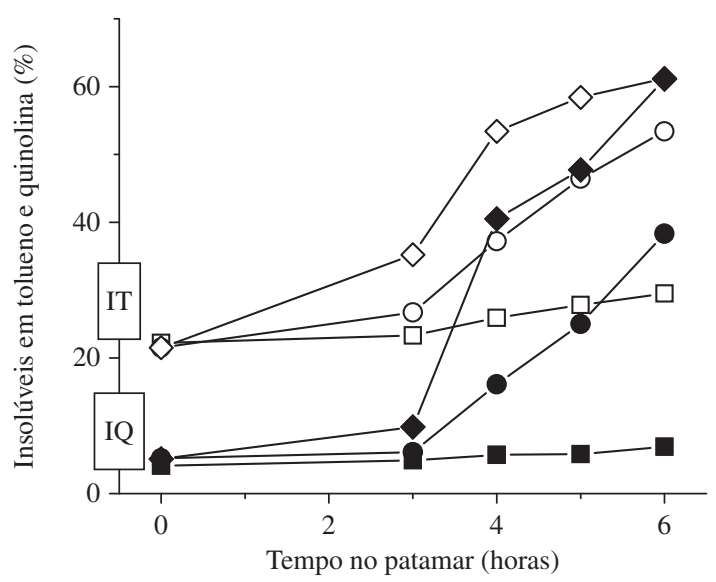

Figura 1. Variação do teor de insolúveis em tolueno (símbolos abertos) e em quinolina (símbolos fechados) como função do tempo de tratamento térmico nos patamares de temperatura de $390{ }^{\circ} \mathrm{C}$ (quadrados), $410{ }^{\circ} \mathrm{C}$ (círculos) e $430^{\circ} \mathrm{C}$ (losangos), para o piche B, sob dois estágios de pressão. o primeiro estágio, que provocou a saída dos componentes mais leves e acelerou a formação da mesofase.

Nessas corridas, realizadas com dois estágios de pressão com o piche isotrópico $\mathrm{B}$, o efeito do aumento da temperatura sobre o ponto de amolecimento foi marcante. A Figura 2 mostra que a variação do ponto de amolecimento dos produtos resultantes do tratamento térmico a $390{ }^{\circ} \mathrm{C}$ foi inferior à variação observada quando a temperatura foi de $410{ }^{\circ} \mathrm{C}$. Valores bem mais elevados de ponto de amolecimento foram determinados para as amostras, que foram submetidas ao aquecimento a $430{ }^{\circ} \mathrm{C}$.

Já com relação ao piche denominado A, a Figura 3 mostra o efeito da temperatura sobre o teor de insolúveis para as amostras recolhidas após o tratamento térmico em dois estágios. Diferentemente do comportamento observado para o piche B, o teor de insolúveis determinado para as amostras obtidas a partir dos tratamentos térmicos a 390 e a $410{ }^{\circ} \mathrm{C}$ sofreu ligeiro aumento. Aumento significativo do teor de insolúveis em tolueno e em quinolina foi observado apenas para as amostras que foram submetidas ao tratamento térmico a $430{ }^{\circ} \mathrm{C}$.

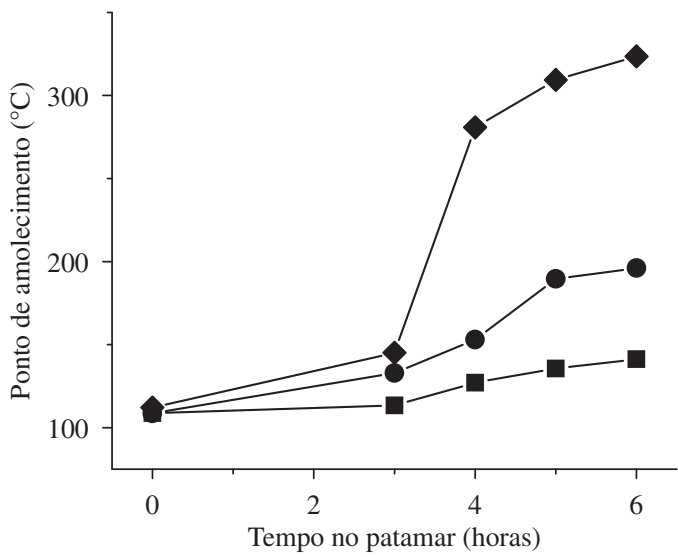

Figura 2. Variação do ponto de amolecimento com o tempo de tratamento térmico do piche $\mathrm{B}$, em dois estágios de pressão, nos patamares de temperatura de $390^{\circ} \mathrm{C}(\boldsymbol{\square}) ; 410^{\circ} \mathrm{C}(\bullet)$; e $430{ }^{\circ} \mathrm{C}(\diamond)$.

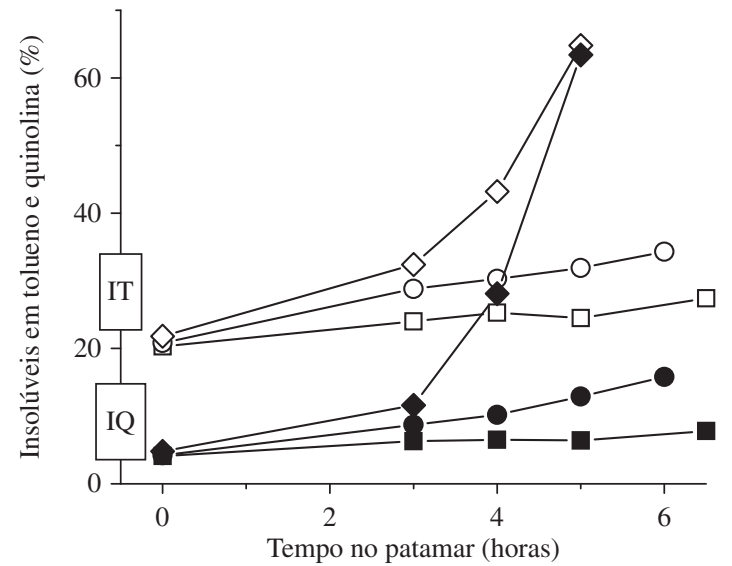

Figura 3. Variação do teor de insolúveis em tolueno (símbolos abertos) e em quinolina (símbolos fechados) como função do tempo de tratamento térmico nos patamares de temperatura de $390{ }^{\circ} \mathrm{C}$ (quadrados), $410{ }^{\circ} \mathrm{C}$ (círculos) e $430{ }^{\circ} \mathrm{C}$ (losangos), para o piche A, sob dois estágios de pressão. 
O mesmo efeito da temperatura pode ser visualizado na Figura 4 para a variação do ponto de amolecimento das amostras recolhidas ao longo do tempo, após os tratamentos térmicos a 390,410 e a $430{ }^{\circ} \mathrm{C}$ do piche isotrópico A. Pode ser verificado que, principalmente após a retirada da pressão, as amostras que sofreram tratamento térmico a $430{ }^{\circ} \mathrm{C}$ apresentaram pontos de amolecimento bem mais elevados, com aumento exponencial em função do tempo.

A Figura 5 mostra a variação do teor de insolúveis em tolueno e em quinolina para as amostras coletadas após os tratamentos térmicos do terceiro piche estudado, C. Nesse caso, o aumento do teor em insolúveis também aumentou com a temperatura e com o tempo. No entanto, o aumento observado com o tratamento térmico a $430{ }^{\circ} \mathrm{C}$ não foi tão significativo como aquele determinado para o piche $\mathrm{A}$, o qual apresentou valores de insolúveis em tolueno (IT) de $65 \%$ após 5 horas de tratamento térmico nesse patamar e de $64 \%$ de insolúveis em quinolina (IQ), enquanto que o piche $\mathrm{C}$ chegou a $58,1 \%$ de IT e 45,8\% de IQ, após o mesmo tempo de patamar.

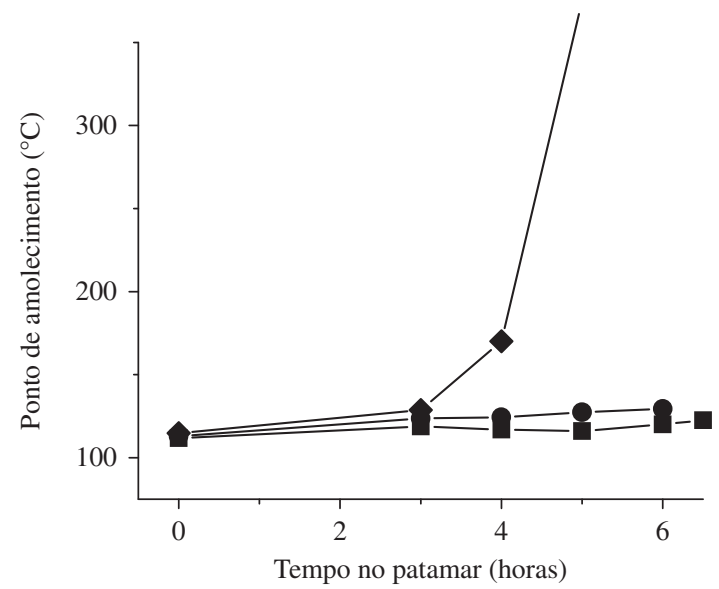

Figura 4. Variação do ponto de amolecimento com o tempo de tratamento térmico do piche $\mathrm{A}$, em dois estágios de pressão, nos patamares de temperatura de $390^{\circ} \mathrm{C}(\boldsymbol{\bullet}) ; 410^{\circ} \mathrm{C}(\bullet)$; e $430^{\circ} \mathrm{C}(\bullet)$.

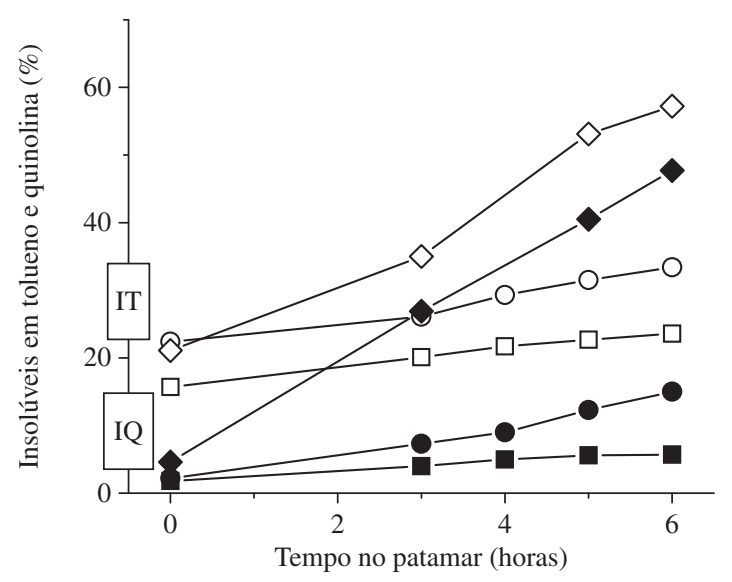

Figura 5. Variação do teor de insolúveis em tolueno (símbolos abertos) e em quinolina (símbolos fechados) como função do tempo de tratamento térmico nos patamares de temperatura de $390{ }^{\circ} \mathrm{C}$ (quadrados), $410{ }^{\circ} \mathrm{C}$ (círculos) e $430{ }^{\circ} \mathrm{C}$ (losangos), para o piche $\mathrm{C}$, sob dois estágios de pressão.

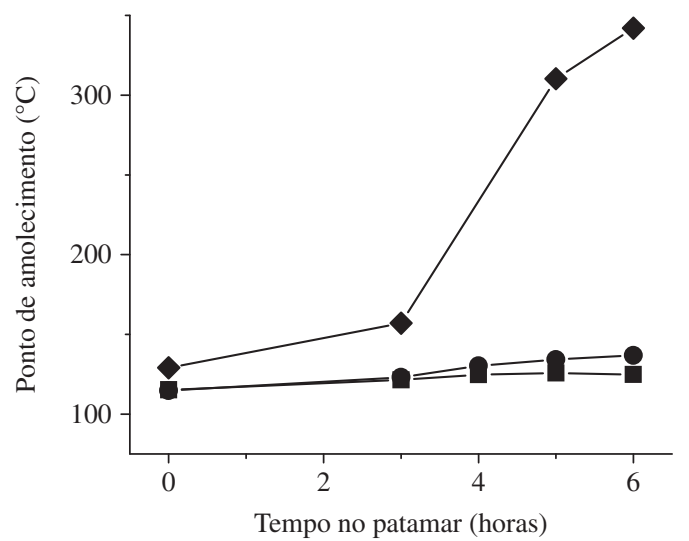

Figura 6. Variação do ponto de amolecimento com o tempo de tratamento térmico do piche $\mathrm{C}$, em dois estágios de pressão, nos patamares de temperatura de $390{ }^{\circ} \mathrm{C}(\boldsymbol{\square}) ; 410{ }^{\circ} \mathrm{C}(\bullet)$; e $430{ }^{\circ} \mathrm{C}(\bullet)$.

Quanto ao ponto de amolecimento (PA) das amostras coletadas a partir da pirólise do piche C, a Figura 6 mostra que tratamentos térmicos, ao longo do tempo, a 390 e a $410{ }^{\circ} \mathrm{C}$, contribuíram para a pequena elevação destes valores, não ultrapassando os $130^{\circ} \mathrm{C}$, após cinco horas de tratamento térmico. Quando o aquecimento foi realizado a $430{ }^{\circ} \mathrm{C}$, o valor do PA chegou a $325^{\circ} \mathrm{C}$, em um período de tempo equivalente.

Quanto ao percentual de mesofase dos piches obtidos após o tratamento térmico por 6 horas no patamar de $430{ }^{\circ} \mathrm{C}$, foram medidos valores de 69 e $56 \%$ para os piches B e C, respectivamente, e de $59 \%$ para o piche A, após 5 horas na mesma temperatura. Nas temperaturas mais baixas, apenas o piche $\mathrm{B}$ apresentou um valor significativo de $44 \%$ de mesofase após 6 horas a $410{ }^{\circ} \mathrm{C}$, enquanto os demais ficaram com valores abaixo de $20 \%$.

\section{Conclusão}

Dentro do tempo de reação máximo de 6,5 horas, durante o qual as experiências foram realizadas, foi verificado que, para todos os piches produzidos, nas temperaturas de 390 e $410{ }^{\circ} \mathrm{C}$ a variação dos parâmetros físico-químicos foi muito pequena, com um comportamento um pouco mais reativo para o piche $\mathrm{B}$, a $410{ }^{\circ} \mathrm{C}$. O ponto de amolecimento ficou sempre abaixo de $200{ }^{\circ} \mathrm{C}$. Para os três piches isotrópicos, os tratamentos térmicos no patamar de $430{ }^{\circ} \mathrm{C}$ produziram piches com pontos de amolecimento acima de $300{ }^{\circ} \mathrm{C}$, e percentual de mesofase de até $69 \%$. Esse resultado indica que, já nesta temperatura, as reações de condensação foram dominantes. Uma comparação dos resultados obtidos a partir dos três piches isotrópicos mostra que o piche B mostrou-se o mais reativo sob todas as temperaturas de reação, com uma aceleração dos aumentos dos parâmetros físico-químicos no segundo estágio do tratamento térmico. Este comportamento do piche B revela a existência de compostos de baixa massa molar, que foram retidos no sistema no primeiro estágio do tratamento térmico e inibiram as reações de condensação que praticamente só passaram a ocorrer após o decréscimo da pressão, no segundo estágio. Os estudos realizados mos- 
traram a possibilidade da produção de piches mesofásicos destinados, dentre outras aplicações, à produção de fibras de carbono, devido ao seu elevado percentual de mesofase. A influência dos parâmetros de reação sobre as propriedades dos piches obtidos foi analisada para posterior correlação com as propriedades mecânicas das fibras a serem obtidas.

\section{Agradecimentos}

Os autores agradecem à Petrobras e à FINEP (CONVÊNIO: 01.04.0586.00 REF. 0492/04) pelo apoio financeiro a esse trabalho.

\section{Referências Bibliográficas}

1. Rand, B.; Hosty, A. J \& West, S. - "Physical Properties of Pitch Relevant to the Fabrication of Carbons Materials", in: Introduction to Carbon Science, H. Marsh (ed.), Butterworth, London, (1989).

2. Edie, D. D. \& McHugh, J. J. - "High Performance Carbon Fibers", in: Carbon Materials for Advanced Technologies; Timothy D. Burchell (ed.), Oak Ridge, (1999).

3. Shimpei, G.; Tomio, A.; Fumio, M.; Kunio, M. and S. Otani - "Process for the preparation of carbon fibers", Carbon, 24, p.11, (1986).
4. Singer, L. S. - "Carbon fibres from mesophase pitch", Fuel, 60, p.839, (1981).

5. Chwastiak, S. - "Low molecular weight mesophase pitch". British Patent Application, GB 2005298A, (1979).

6. Riggs, D. M. \& Diefendorf, R. J. - "Solvent extracted pitch precursors for carbon fibers", in: $16^{\text {th }}$ American Carbon Conference, American Carbon Society, Extended Abstracts, San Diego, p.24 (1983).

7. Yamada, Y. \& Honda, H. - "Preparation of carbon fiber", Japan Patent 58-18421 (1983).

8. Park, Y. D. \& Mochida, I. - "A two-stage preparation of mesophase pitch from the vacuum residue of FCC decant oil", Carbon, 27, p.923, (1989).

9. Oh, S.; Yoon, S. H.; Dong, G. \& Park, Y. D. - "Effects of pressurized pretreatment on the preparation of mesophase pitch", Carbon, 29, p.1009 (1991).

10. Dutra, C. H. M. C.; Freitas, L. C. \& Franceschi, F. - "Determinação do Conteúdo de Mesofase em Piches por Centrifugação", in: Anais do IV Congresso Brasileiro de Carbono - Carbono 2007- Gramado-RS - nov. - p.20 - 1 CD-ROM (2007).

Enviado: 11/03/08

Reenviado: 30/05/08

Aceito: 02/06/08 